\title{
IDOSOS COM DOENCA DE ALZHEIMER E FAMÍLIA: UMA PROPOSTA DE PRÁTICA GERONTOLÓGICA NA PROMOÇÃO DE VÍNCULOS E COMUNICAÇÃO
}

\author{
Henrique Salmazo da Silva ${ }^{1}$ \\ Flávia Renata Fratezi² \\ Andrea Lopes ${ }^{3}$
}

resumo

O objetivo deste estudo foi avaliar uma intervenção gerontológica planejada para favorecer o fortalecimento de vínculos e a comunicação entre familiares e idosos portadores da Doença de Alzheimer (DA), residentes em uma Instituição de Longa Permanência para Idosos (ILPI), situada no município de São Paulo. Participaram do

1 Bacharel em Gerontologia pela Escola de Artes, Ciências e Humanidades da Universidade de São Paulo (EACH/USP). Mestre em Ciências pela Faculdade de Saúde Pública da Universidade de São Paulo (USP). Doutorando em Neurociências e Cognição pela Universidade Federal do ABC (UFABC). Instituição: EACH/USP. E-mail: henriquesalmazo@yahoo.com.br

2 Bacharel em Gerontologia pela Escola de Artes, Ciências e Humanidades da Universidade de São Paulo (EACH/USP). Pesquisadora do Laboratório de Inovação em Planejamento, Gestão, Avaliação e Regulação de Políticas, Sistemas, Redes e Serviços de Saúde do Instituto de Ensino e Pesquisa do Hospital do Coração (LIGRESS/IEP/HCor). Instituição: EACH/USP. E-mail: flaviafratezi@gmail.com

3 Antropóloga. Mestre em Gerontologia e Doutora em Educação/Gerontologia. Docente do curso de Graduação em Gerontologia da Escola de Artes, Ciências e Humanidades da Universidade de São Paulo (EACH/USP). Coordenadora dos grupos de pesquisa EAPIS-EACH USP e ENVOLVE EACHUSP. E-mail: andrealopes@usp.br 
estudo três idosos e três familiares (sobrinhas e filhas). As atividades consistiram em cinco encontros temáticos para os familiares, com duração de 90 minutos cada e um encontro de integração entre familiares e idosos. Os encontros abordaram as alterações que acompanham a DA, as representações sobre a institucionalização e a confecção de um álbum de fotografias composto por fotos de cada idoso ao longo do seu curso da vida, material usado como uma ferramenta para resgatar suas histórias de vida. A utilização do álbum no encontro de integração possibilitou a promoção da comunicação e o fortalecimento do vínculo afetivo entre os idosos e seus familiares. Ao final da intervenção, os familiares relataram que os encontros propiciaram a troca de experiências, a obtenção de mais conhecimento sobre a doença e a aproximação com o idoso. Observou-se que as atividades potencializaram o nível de interação entre os familiares e os idosos.

palavras - chave

Idoso. Institucionalização. Doença de Alzheimer. Intervenção.

\section{Introdução}

Em face de situações de dependência e perda da autonomia do idoso, muitas famílias não possuem condições de prover os cuidados a essas pessoas, necessitando do auxílio de serviços especializados e de ajuda profissional. Em revisão de literatura, Zarit (2009) descreveu que as enfermidades que representam maior fonte de ônus e sobrecarga para os cuidadores são as neurodegenerativas, como a Doença de Alzheimer (DA), que ocasionam no portador declínio das habilidades funcionais, alterações comportamentais e perda dos dados autobiográficos, principalmente nos estágios moderados da doença.

Nesse contexto, as Instituições de Longa Permanência para Idosos (ILPI) são os principais serviços que proveem cuidados aos idosos fora do ambiente familiar (Christophe e Camarano, 2010). Trata-se de instituições de caráter residencial coletivo, governamentais ou não-governamentais, destinadas a acolher pessoas com idade igual ou superior a 60 anos, com ou sem suporte familiar, em condições de liberdade, dignidade e cidadania (ANVISA, 2005; BORN; BOECHAT, 2006; CAMARANO; MELLO, 2010). 
Dentre as razões para a institucionalização, encontra-se, principalmente, o esgotamento das possibilidades familiares de cuidado (KARSCH, 2003). Nessa circunstância, a atenção à família configura-se como uma dimensão importante para o sucesso do ingresso do idoso na ILPI, devendo os profissionais envolvidos auxiliar os familiares a manejar pressões emocionais, afetivas e externas (econômicas, culturais) relacionadas ao cuidado (CORTELLETTI et al., 2010; NORRIS, 2009; QUEROZ, 2010; ZARIT, 2009), bem como administrar as mudanças diversas, especialmente vinculares, que são subjacentes a esse processo. O esgotamento das possibilidades de cuidado, aliado ao estresse físico e mental do cuidador são aspectos que precisam ser trabalhados (ZARIT, 2009). Se considerarmos que a família é uma das principais fontes de suporte social do idoso, torna-se importante refletir sobre o impacto da institucionalização no sistema familiar e no bem-estar do idoso (ANTONUCCI et al., 2001; NORRIS, 2009).

Elliot e colaboradores (2007), ao estudarem grupos de cuidadores informais norte-americanos de idosos acometidos pela DA, revelaram que os participantes sentiam dificuldades em manejar a perda da autonomia do idoso, exibida em estágios intermediários da doença e na tomada de decisões a respeito do cuidado direcionado aos pais e familiares. O estudo indicou que a transferência do familiar para a própria residência e a posterior institucionalização levaram a limiares de estresse excessivamente altos nesses cuidadores. Observou-se que a negociação dos cuidados envolveram conflitos e a abertura de canais de diálogo entre os diferentes membros, que vivenciaram uma nova etapa do ciclo de vida familiar, incluindo a redefinição de papéis.

Sobre esse aspecto, Zarit (2009) indicou que o declínio na capacidade funcional do idoso solicita a reorganização do sistema familiar e exige cuidados e atenção. Assim, compreender a história de vida familiar e as fases que os membros vivenciam é importante para resgatar o significado de como as doenças, em especial a DA, podem impactar na dinâmica familiar e na vida de cada membro.

Envolver a família no cotidiano da ILPI e fortalecer o vínculo entre idosos portadores de DA e seus familiares constitui um desafio (NORRIS, 2009). Por um lado, o Estatuto do Idoso em seu Artigo 49 sugere a preservação dos vínculos familiares pelas instituições de longa permanência (BRASIL, 2003). Por outro, Davim et al. (2004) indicaram que as ILPIs podem favorecer a inatividade física e mental do idoso e a ruptura dos laços sociais, levando ao seu isolamento social.

Segundo Norris (2009) e Queroz (2010), os profissionais devem trabalhar para que a institucionalização não simbolize o rompimento de vínculos 
afetivos e o término das relações de cuidado. Programas desenvolvidos com familiares e cuidadores destacam a importância de se veicular informações sobre a saúde do idoso, refletir sobre os métodos de enfrentamento do estresse relacionado ao cuidado e desmistificar os mitos a respeito da institucionalização, oferecendo alternativas para a inserção da família no cuidado (CORTELLETTI et al. 2010; DUCHARME et al., 2005; NORRIS, 2009; QUEROZ, 2010; ZARIT, 2009).

Assim, o objetivo deste trabalho foi avaliar uma intervenção gerontológica, planejada para favorecer o fortalecimento de vínculos e a comunicação entre três idosos portadores de DA, residentes em uma ILPI situada no município de São Paulo, e seus familiares.

\section{Método}

O projeto tratou de um estudo qualitativo e de intervenção gerontológica, desenvolvido por graduandos do oitavo semestre do curso de graduação em Gerontologia em uma ILPI pública, no município de São Paulo, entre os meses de agosto e dezembro de 2008. Por se tratar de uma ILPI mista (pública e filantrópica), parcela dos idosos assistidos era encaminhada por meio do Ministério Público e pela Secretaria Estadual de Assistência Social de São Paulo, acolhendo casos de abusos, maus-tratos, negligência, fragilidade de vínculos familiares e insuficiência familiar na promoção dos cuidados. A ILPI atendia 56 idosos e apresentava como objetivo oferecer atendimento social e de enfermagem no âmbito da Proteção Social de Alta Complexidade do Sistema Único da Assistência Social (SUAS), tendo em seu quadro de funcionários: assistente social, enfermeira, auxiliares de enfermagem, gerente, profissionais da cozinha e limpeza. A instituição foi escolhida para a realização da pesquisa por ter sido campo de estágio para os graduandos e por ter acolhido a proposta deste estudo.

Considerando que, do total de 56 idosos residentes na ILPI, grande proporção era de portadores da DA em estágio inicial e moderado, a intervenção gerontológica ora tratada, surgiu da necessidade de propor atividades para esse público. As atividades foram planejadas com o objetivo de manter e estimular as capacidades que ainda estavam preservadas nos idosos, especialmente através do vínculo familiar de boa qualidade.

Todos os familiares dos idosos portadores de DA institucionalizados foram convidados a participar do estudo. Utilizou-se, como estratégia para os convites, o contato telefônico e o contato presencial nos dias de visita e festas 
da ILPI. Os encontros, segundo regimento da ILPI, só poderiam ser feitos durante a semana e nos dias de visita e horários já estabelecidos pelo serviço.

Diante desses fatos, três familiares apresentaram disponibilidade para participar, o que demandou a adaptação da proposta inicial e permitiu o desenvolvimento de encontros direcionados para o contexto de cada família assistida. Os três familiares eram mulheres, sendo uma filha e duas sobrinhas dos idosos, com escolaridade igual, ou superior ao ensino médio, idade entre 35 e 55 anos, sendo duas casadas e uma solteira. As participantes visitavam seus familiares pelo menos uma vez por semana. Esse foi um dos fatos que possibilitou a participação delas nos encontros temáticos.

Os critérios para inclusão na intervenção compreenderam: ter disponibilidade para comparecer à instituição nas datas programadas, sendo essa uma normativa da ILPI em que o estudo foi desenvolvido; ser familiar de idosos residentes na ILPI portadores da doença de Alzheimer na fase moderada, possuir fotografias do idoso e sua família; e ser idoso portador de doença de Alzheimer na fase moderada, residente na ILPI, cujo familiar tenha aceitado participar do estudo.

A participação somente de familiares de idosos portadores da doença de Alzheimer no trabalho foi uma demanda da própria ILPI, visto que, como dito anteriormente, uma grande maioria dos residentes possuía a doença. Os funcionários da instituição não foram convidados a participar do estudo, uma vez que, por se tratar de uma intervenção com familiares, considerou-se que a presença de funcionários poderia inibir a participação da família.

A intervenção gerontológica consistiu em cinco encontros temáticos, com duração de 90 minutos cada, sendo quatro para os familiares e um encontro de integração entre familiares e idosos. Os encontros que envolveram os familiares tiveram como objetivos (1) transmitir conhecimentos a respeito da DA e do processo de envelhecimento; (2) discutir as representações sobre a institucionalização e as ILPIs; e (3) capacitar os familiares para a montagem de um álbum de fotografias, composto por fotos do idoso ao longo do seu curso de vida. Por fim, (4) o encontro entre o idoso e o familiar organizou-se na promoção desse vínculo através da manipulação do álbum de fotografias.

Os temas discutidos nos encontros incluíram: mitos e estereótipos sobre o envelhecimento, discutindo o papel do idoso na sociedade; a estrutura e a dinâmica familiar antes e depois da institucionalização do idoso, abordando a qualidade das relações familiares e o histórico da família antes da institucionalização; os significados atribuídos à institucionalização e à ILPI, incluindo as pressões internas e externas sofridas pela família frente aos déficits cognitivos e às demandas relacionadas ao cuidado; a doença de 
Alzheimer no contexto familiar e institucional, discutindo possibilidades de intervenção e cuidado; reflexões sobre como os familiares poderiam contribuir para as atividades desenvolvidas na instituição e, ainda, realizar ações que objetivassem o bem-estar do idoso e o fortalecimento dos laços afetivos entre os idosos e a rede familiar.

Esse método de trabalho foi planejado com o objetivo de preparar os familiares para a intervenção com os idosos, estimulando a promoção dos vínculos e de possibilidades efetivas de comunicação. Os encontros basearam-se nos programas psicoeducativos e terapêuticos desenvolvidos para familiares de idosos com DA, cujos objetivos são oferecer suporte emocional, trocar informações, construir conhecimentos, compartilhar dilemas e aprender maneiras de manejar estressores internos e externos relacionados ao cuidado (DUCHARME et al., 2005; ZARIT, 2009).

Para incentivar a participação dos familiares, foram utilizadas dinâmicas e estratégias pedagógicas como músicas, poemas, vídeos e materiais explicativos sobre a DA, confeccionados pela Associação Brasileira de Alzheimer (ABRAz).

$\mathrm{O}$ álbum de fotografias foi utilizado no encontro de integração entre familiares e idosos. A proposta do álbum surgiu em face à possibilidade de estimular o idoso a resgatar dados autobiográficos e de estimular a família a interagir com o idoso, revestindo significados à visita. A proposta, como foi desenvolvida, privilegiou os familiares que já tinham algum tipo de vínculo com os idosos. Segundo Zarit (2009), intervenções psicológicas, terapêuticas e no histórico de vida das famílias exigem tempo e investimento. Por essa razão, em face de escassez de tempo, optou-se por uma intervenção mais simples e que pudesse otimizar e manter o vínculo dos familiares já presentes na ILPI no período de realização do estágio, sem ocasionar prejuízo no objetivo do estudo.

Para a confecção dos álbuns, os familiares foram instruídos a selecionar as fotos que simbolizavam eventos importantes para o idoso, como casamentos, batizados, passeios, festas e encontros de família. Priorizaram-se fotos que estimulavam alegria e memórias agradáveis, visto que o estado de humor do idoso com DA associa-se ao seu nível de qualidade de vida e ao conteúdo afetivo das memórias evocadas (ZARIT, 2009). Na ocasião da apresentação do álbum aos idosos, os familiares foram orientados a iniciar pelas fotos mais antigas, seguindo para as mais recentes, aumentando as possibilidades de resgate e evocação dos dados autobiográficos pelo idoso. Essa estratégia foi usada, tendo em vista que os dados autobiográficos são perdidos ao longo da doença, permanecendo as lembranças mais antigas e significativas (CANINEU, 2002; NORMURA et al., 2000). 
Para o desenvolvimento da intervenção e avaliação da sua efetividade, utilizaram-se técnicas próprias do método etnográfico, incluindo: (a) observação livre e participante das atividades oferecidas pela ILPI, devidamente acompanhada por registro em caderno de campo, executadas especialmente no início do estudo para compreensão do universo pesquisado; (b) entrevista em profundidade junto aos familiares no primeiro contato realizado, com o objetivo de resgatar o histórico da família e as transições agenciadas antes da institucionalização do idoso (vide Anexo - Roteiro Semi-estruturado de Entrevista I); (c) Entrevista dirigida através de um questionário com perguntas abertas e fechadas sobre a percepção acerca da DA, institucionalização e envelhecimento, o qual foi aplicado no início e ao final da intervenção (vide Anexo - Questionário II). Ambos os instrumentos foram elaborados com base na literatura científica sobre o tema, especialmente nas propostas de intervenção desenvolvidas para familiares de idosos portadores da DA (Falcão, 2008). Tanto nas entrevistas, como no questionário, buscou-se também ressaltar os aspectos relevantes para a proposta obtidos nas observações. Todos os encontros foram gravados e fotografados, com o consentimento dos participantes e da instituição, material que serviu de registro da experiência.

O tratamento e análise dos dados acompanharam as orientações do método etnográfico, que tem como proposta estabelecer relações, caracterizar o meio e interpretar os significados dos códigos e da lógica de funcionamento do grupo investigado o que, conforme Geertz (1978), podemos chamar de teia de relações. A literatura especializada sobre o assunto também foi consultada (CAMARANO, 2010; DUCHARME et al., 2005; NORRIS, 2009; QUEROZ, 2010; ZARIT, 2009).

Os participantes assinaram o Termo de Consentimento Livre e Esclarecido, segundo as diretrizes do MS 196/96 e de acordo com a supervisão de uma equipe de docentes do curso de Gerontologia da EACH/USP. Houve também comunicação e anuência, por parte da equipe de profissionais e responsáveis pela ILPI, através da assinatura de um Termo Institucional de Consentimento Livre e Esclarecido.

\section{Resultados e Discussão}

As respostas dos participantes ao questionário aplicado no início da intervenção indicaram que, na visão dos familiares, a institucionalização e a ILPI possuíam um caráter de abandono, provavelmente associado a influências socioculturais de que a família deve ser a principal provedora dos 

1999; QUEROZ, 2010). Para Cortelletti et al. (2010), as ILPIs no Brasil são relacionadas à pobreza, negligência e abandono do idoso pelas famílias, o que gera nos familiares sentimentos de culpa, negação e fracasso.

De acordo com as familiares, segundo o questionário e entrevistas, o processo de institucionalização foi um momento doloroso para as famílias, mas que se configurou como uma solução para parte de seus problemas, como a sobrecarga de atividades e a escassez de tempo para prover os cuidados necessários. Embora tivessem vínculos afetivos preservados, não conseguiam conciliar a tarefa do trabalho e da vida familiar com aquelas relacionadas aos cuidados aos idosos. Dados semelhantes foram relatados na literatura (CAMARANO, MELLO, 2010; CORTELLETTI et al. 2010; ELLIOT et al., 2007; KARSH, 2003; NERI, SOMMERHALDER, 2002).

A relação de afeto que as participantes tinham com os idosos dificultou a aceitação da separação, ocasionando ônus e sofrimento psíquico. A decisão pela institucionalização surgiu em face do curso da DA, pois as famílias não estavam preparadas para lidar com a situação. É possível que esse fator tenha influenciado a não participação dos outros familiares na intervenção, uma vez que a intervenção não contou com um trabalho prévio de sensibilização e educação. Face ao rompimento dos vínculos afetivos e das situações de abandono e negligência nas quais se encontravam muitos idosos, é possível que um trabalho de prevenção e promoção de vínculos intrafamiliares, ainda no âmbito familiar, tenha maior ressonância, antes que a família se sinta sobrecarregada e sozinha ao prover os cuidados, optando pela institucionalização. Karsch (2003) descreveu que, no Brasil, a decisão pela institucionalização acontece principalmente quando a família chega ao limite da sua capacidade em oferecer os cuidados necessários. A decisão, via limite dos recursos diversos, pode ocasionar possível dificuldade futura de manutenção dos laços afetivos.

As participantes relataram que, no momento da institucionalização, os idosos não foram consultados sobre a decisão, o que gerou nos familiares um sentimento de culpa. Apesar disso, acreditaram ter sido a melhor escolha, por considerarem que não possuíam suporte emocional, social, físico, psicológico e financeiro, nem conhecimento adequado para cuidarem dos idosos em suas residências. Dados semelhantes foram encontrados por Almeida (2005), ao entrevistar oito idosos institucionalizados, que relataram que seus familiares, amigos ou médicos mediaram à ida para a instituição e que em nenhum dos casos a iniciativa partiu do idoso. Conforme sinalizado anteriormente, esses dados indicam que intervenções gerontológicas devem ser iniciadas no momento que antecede a institucionalização, com o objetivo de 
auxiliar os familiares, idosos e profissionais na adaptação e entrada na ILPI (CORTELLETTI et al., 2010).

As reflexões das participantes no questionário inicial e nas questões abordadas durante os encontros indicaram que o sistema familiar sofreu alteração após a institucionalização dos idosos, principalmente pela diminuição dos conflitos familiares. Nesse contexto, a família é um sistema em interação com o ambiente, composta por subsistemas, que são os seus membros. Os subsistemas estão em interação, da qual resulta a organização do sistema familiar (FALCÃO, 2006; FALCÃO, 2010). Alterações de qualquer natureza, seja física, psicológica, ou social, em um subsistema, afeta os demais e modifica toda a estrutura e a dinâmica familiar. Assim, o portador de DA exibe comportamentos que afetam os demais membros da família, pois esses, em geral, não entendem e não aceitam tais comportamentos, ou mesmo a mudança de suas rotinas.

Além disso, os portadores da DA possuem uma hierarquia e um papel dentro da família, que são modificados devido à doença, uma vez que eles não possuem mais discernimento para exercer qualquer tipo de poder, ou papel no sistema familiar. Nesse sentido, a família tem que se adaptar para que novas relações de poder e, muitas vezes, manutenção financeira, sejam ressignificadas e estabelecidas, exigindo que outros membros assumam os papéis que antes pertenciam ao portador da doença (ELLIOT et al., 2007; FALCÃO, 2006; ZARIT, 2009).

Ao discutir informações a respeito das alterações que acompanham a DA, as participantes relacionaram os conteúdos à experiência pela qual haviam passado com os idosos, buscando compreender o motivo de determinados comportamentos (CANINEU, 2002; NOMURA et al., 2000). As participantes relataram terem observado nos idosos: perda progressiva da memória, desorientação visuoespacial, ideias delirantes, apatia, agressividade e diminuição da capacidade intelectual.

As familiares chegaram ao consenso de que a atenção ao idoso com DA requer uma atuação interprofissional e compreenderam o comportamento dos idosos da ILPI portadores da doença, comentando situações como: um dia em que encontraram um idoso sem calças na entrada da instituição; o fato de seus familiares falarem que alguém pegou o dinheiro que haviam guardado; a dificuldade em manter um diálogo com alguns idosos da ILPI; a marcha arrastada; a agitação noturna que perceberam em seus familiares, dentre outras. (CANINEU, 2002).

No quinto e último encontro, em que os familiares apresentaram o álbum confeccionado aos idosos, todos os idosos reconheceram-se nas foto- 
grafias e resgataram alguns eventos de vida durante a exposição do álbum. Um dos idosos, na ocasião da exposição do álbum, resgatou a lembrança de uma das participantes como sendo sua filha, fazendo uma relação entre ela e as fotografias em que aparecia. Outra idosa evocou em uma das fotos seu esposo e namorado, relatando à sobrinha que ele havia sido o amor de sua vida, indicando que as fotos propiciaram a evocação de dados autobiográficos importantes para a manutenção do histórico de vida e das referências pessoais da idosa.

Estudos indicam que intervenções baseadas nas reminiscências de vida podem otimizar o desempenho de memória e a afetividade dos idosos (CANINEU, 2002; ZARIT, 2009). No contexto dos idosos com DA, a manutenção dos dados autobiográficos em fases moderadas da doença é recomendável e deve ser estimulada pelos profissionais (CANINEU, 2002).

Como mencionado, cada familiar foi orientado a mostrar primeiro as fotografias mais antigas e, posteriormente, as mais recentes, fazendo perguntas que estimulassem os idosos a elaborar narrativas sobre as fotos (NOMURA et al., 2000). Após a atividade, foi destacado que a apreciação do álbum deveria ocorrer com regularidade para que se observasse, através da sistematização, uma melhora no resgate da informação pelo idoso. Porém, foi explicado que nem sempre os idosos iriam lembrar coisas que haviam lembrado anteriormente; que eles poderiam se lembrar de fatos que não lembraram antes; e que deveria ser valorizado o momento de exposição do álbum e contado com o material biográfico do álbum, como forma de promoção de vínculo, independente do fato de o idoso não se lembrar do que aconteceu, minutos depois.

O questionário de avaliação, ao final do estudo, revelou que as familiares possuíam conhecimento prévio sobre a DA e suas características, o qual foi aprimorado durante os encontros do grupo. Com relação aos sentimentos perante a institucionalização, as familiares disseram que, com os encontros, passaram a ter sentimentos mais positivos, com diminuição dos sentimentos de culpa, abandono e impotência. Embora essa avaliação tenha se baseado em um questionário semiestruturado, e não tenham sido aplicadas escalas de avaliação de sintomas depressivos, ansiedade e bem-estar psicológico pré e pós intervenção, esses dados estão em consonância com outros estudos que desenvolveram intervenções psicoeducacionais com familiares de idosos portadores de DA (DUCHARME et al., 2005; NORRIS, 2009; QUEROZ, 2010; ZARIT, 2009).

Segundo Zarit (2009), a avaliação da eficácia dessas intervenções ainda é difícil de ser mensurada, uma vez que são desenvolvidas em pequenos grupos e com desenhos diferentes: perfil do familiar, sobrecarga dos cuidados, 
temas desenvolvidos, número de sessões, entre outros. No contexto das ILPIs, o desafio dos profissionais gerontólogos baseia-se em transformar o apoio familiar em apoio emocional e que possa ser gratificante para o idoso e família, rompendo os processos de afastamento, isolamento e abandono familiar (NORRIS, 2009).

Além disso, as respostas mostraram que as familiares amadureceram a ideia de que a relação com os idosos poderia melhorar mesmo diante da DA, sendo importante compreender e respeitar os limites e condição do idoso.

Com relação ao tema envelhecimento e velhice abordado em um dos encontros, percebeu-se que, ao final da intervenção, o envelhecimento passou a ser mais associado a uma condição natural da vida. Houve a noção de que o "saber envelhecer" é uma atitude que acompanha o indivíduo por todo o seu ciclo de vida. Já a velhice passou a ser entendida como a última fase do ciclo de vida.

Ao final, as familiares relataram que os encontros possibilitaram trocar experiências, entender e aceitar a doença, obter mais conhecimento sobre a DA e possibilitar a proximidade com o familiar idoso. Esses resultados estão em consonância com as intervenções grupais e psicoeducacionais desenvolvidas com cuidadores familiares, que ampliam as possibilidades de participação, troca de informações, discussão sobre a resolução de pressões emocionais e estressores, levantamento das possibilidades de inserção e engajamento da família (DUCHARME et al., 2005; ZARIT, 2009). Segundo Norris (2009) a atenção à família torna-se fundamental, pois a saúde mental dos idosos institucionalizados é afetada pelos vínculos familiares.

O presente estudo com o grupo de familiares de idosos residentes na ILPI possibilitou o resgate da biografia do idoso, fortalecendo vínculos e a comunicação entre idosos e familiares, por meio do álbum de fotografias; a capacitação das famílias sobre a doença de Alzheimer; a ressignificação do ato de institucionalizar o idoso e a motivação dos familiares a participarem mais da rotina do idoso e da instituição.

\section{Considerações Finais}

Quando a família não tem suporte para lidar com os encargos advindos de uma situação de doença de um membro, formas alternativas de oferecer cuidado e atenção devem ser consideradas, como por exemplo, a institucionalização. Enquanto os cuidados de longa duração ainda são escassos em nosso meio, incluindo os centros-dia, hospitais-dia e assistência domiciliar, 
a ILPI é uma possibilidade viável de apoio, suporte e amparo instrumental e emocional aos idosos e familiares (CAMARANO e MELLO, 2010; CORTELLETTI et al., 2010). Por essa razão, acredita-se que intervenções gerontológicas junto às ILPIs devem assessorar a família e oferecer melhor qualidade de vida a todos os envolvidos.

Observou-se que as atividades potencializaram o nível de interação entre os familiares e os idosos. $\mathrm{O}$ resgate de dados autobiográficos, um dos itens que gradativamente são perdidos ao longo do curso da DA, pode ser uma ferramenta de estimulação utilizada pelos próprios familiares para o estreitamento do vínculo com o idoso.

Uma das limitações desse estudo foi a impossibilidade de continuar os encontros, acompanhando os efeitos da intervenção ao longo do tempo e a qualidade do vínculo que foi estimulado. Outras limitações incluem a dificuldade de envolver outros familiares dos idosos residentes na ILPI, em virtude do horário em que aconteciam as intervenções e dos critérios de participação estabelecidos. A partir dessa experiência, entende-se que a intervenção, quando feita a longo prazo e com supervisão contínua, pode ser ampliada e desenvolvida para idosos e familiares com vínculos menos estreitos, oferecendo suporte psicossocial à família e ao idoso. Neste estudo, a intervenção se restringiu aos idosos com demência, com vínculos mais fortalecidos com familiares e que foram institucionalizados em virtude do ônus associado aos cuidados. O trabalho anterior à institucionalização deve ser ressaltado, estimulando a possibilidade de permanência do idoso, por um maior tempo possível, junto aos familiares e na comunidade.

Acredita-se que estratégias devem ser mobilizadas por profissionais capacitados para envolver o máximo de famílias e idosos, incluindo um trabalho integrado com a equipe técnica e com os profissionais que supervisionam a ILPI.

Diante do perfil de idosos da ILPI e da escassez de recursos físicos e humanos, a implementação do projeto mostrou-se efetiva, pois ao estimular o histórico de vida dos residentes, as visitas das famílias tornaram-se revestidas de novos propósitos e significados, em uma nova fase da vida, por parte de ambos os grupos de envolvidos; ao conhecer mais sobre a doença e sobre os serviços, a família aumentou o arsenal de estratégias para enfrentar as adversidades associadas à doença e ao cuidado ao idoso.

Em outras palavras, os familiares passaram a compreender melhor o cotidiano da instituição, refletindo sobre a abordagem necessária para atender os idosos com DA. Assim, a partir dessa experiência piloto propõese a criação de um grupo organizado e permanente de familiares na ILPI, configurando-se como "Familiares amigos da ILPI". Nesse grupo, os fami- 
liares poderiam trocar experiências, discutir formas de melhorar a atenção ao idoso e otimizar os recursos disponíveis na instituição. Esse grupo inicial já treinado poderia, ainda, gerenciar e recepcionar outros e novos familiares, configurando-se como um grupo de apoio.

\author{
OLDER ADULTS WITH ALZHEIMER'S DISEASE \\ AND FAMILY: A PROPOSAL OF GERONTOLO- \\ GICAL PRACTICE TO PROMOTE TIES AND \\ COMMUNICATION
}

\title{
ABSTRACT
}

The aim of this study was to evaluate an intervention designed to promote gerontological ties and communication between families and older adults with Alzheimer's disease (AD) residing in an Institution of Long Term Care to older adults, located in São Paulo. Participants were three seniors and three relatives (nieces and daughters). The activities consisted of five thematic meetings for family members, lasting 90 minutes and one meeting of integration between family and the older adult. The meetings addressed the changes that accompany the DA, the representations of institutionalization and the production of an album of photos used as a tool to reconstruct the history of life of elderly. The use of the album in the integration meeting contributed to enhancing the communication and strengthens ties between older adult with DA and the families. At the end of the intervention, the family reported that the meetings enabled the exchange of experiences, to obtain more knowledge about the disease and the approach to the elderly. It was observed that the activities potentiated the level of interaction between the families and the elderly.

keywords

Elderly. Institutionalization. Alzheimer's disease. Intervention.

referências

ALCÂNTARA, Adriana de Oliveira. Velhos Institucionalizados e Familia: entre abafos e desabafos. Campinas: Alínea, 2004.

ALMEIDA, Fabiana Souza. Idosos em instituições asilares e suas representações sobre família. Dissertação (Mestrado em Sociologia). Universidade Federal de Goiás, Goiania, 2005. 
ANTONUCCI, Toni. Social Relations: An Examination of Social Networks, Social Support, and Sense of Control. In: BIRREN, James; SCHAIE, K. Warner (Orgs) Handbook of the Psychology of Aging. San Diego: Academic Press, 2001, 5 ed., Cap. 17, p- 427-448.

ANVISA - AGÊNCIA NACIONAL DE VIGILÂNCIA SANITÁRIA - DIRETORIA COLEGIADA RESOLUÇÃO ANVISANDC No 283, DE 26 DE SETEMBRO DE 2005, Diário Oficial da União; Poder Executivo, Brasília, DF, 27 set. 2005. Seção 1, p. 58-59.

BORN, Tomiko; BOECHAT, Norberto. A Qualidade dos Cuidados ao Idoso Institucionalizado. In: FREITAS, Elizabete Viana de et. al (Org.) Tratado de Geriatria e Gerontologia. 2 ed. Rio de Janeiro: Guanabara-Koogan, 2006. p.1131-1141.

BRASIL. Lei no 10.741, de 1 de outubro de 2003. Dispõe sobre o Estatuto do Idoso e dá outras providências. Disponível em: <http://uww.planalto.gov.br>. Acesso em: 10 set. 2008 .

CAMARANO, Ana Amélia; MELLO, Juliana Leitão e. Introdução. In: CAMARANO, Ana Amélia (Org.) Cuidados de longa duração para a população idosa: um novo risco social a ser assumido? Rio de Janeiro: IPEA - Instituto de Pesquisa Econômica Aplicada, 2010. pp-01-10.

CANINEU, Paulo Renato. Doença de Alzheimer. In: CAOVILLA, Vera Pedrosa; CANINEU, Paulo Renato (Orgs.). Você não está sozinho: ela não sabe que sou sua filha, mas eu sei que ela é minha mãe. São Paulo: Abraz, 2002.

CHRISTOPHE, Micheline; CAMARANO, Ana Amélia. Dos asilos às instituições de longa permanência: uma história de mitos e preconceitos. In: CAMARANO, Ana Amélia. (Org.) Cuidados de longa duração para a população idosa: um novo risco social a ser assumido? Rio de Janeiro: IPEA - Instituto de Pesquisa Econômica Aplicada, 2010. p. $145-162$

CORTELlEITI, Ivone; CASARA, Miriam Bonho; HERÉDIA, Vânia Beatriz Merlotti et al, Idoso asilado: um estudo gerontológico. 2 ed. Caxias do Sul, RS: EDIPUCRS, 2010.

DAVIM, Rejane Marie Barbosa; TORRES, Gilson de Vasconcelos; DANTAS, Suzana Maria Miranda; LIMA, Vilma Maria. Estudo com idosos de instituições asilares no município de Natal/RN: características socioeconômicas e de saúde. Revista Latino-Americana de Enfermagem, Ribeirão Preto, v. 12, n. 3, p. 518-524, 2004.

DEBERT, Guita Gren. A Reinvenção da Velhice: Socialização e Processos de Reprivatização do Envelhecimento. São Paulo: Editora da Universidade de São Paulo: FAPESP, 1999.

DUCHARME, Francine, LÉVESQUE, Louise; GIROUX, Francine. Follow-up of a intervention program for caregivers of a relative with dementia living in a long-term care setting: Are there any persistent and delayed effects? Aging \& Mental Health, Abingdon, v. 9, n. 5, p. 461-469, 2005.

ELLIOT, Barbara et al. Decision making on behalf of elders with advanced cognitive impairment - Family transitions. Alzheimer Disease and Associated Disorders, Hagerstown, v. 21, n. 1, p. 49-54, 2007

FALCÃO, Deusivania Vieira da Silva (Org.) A Familia e o idoso: Desafios da contemporaneidade. Campinas, SP: Papirus, 2010

FALCÃO, Deusivania Vieira da Silva. Questionário de percepção sobre doença de Alzheimer. Grupo de Atenção Psicogerontológica e Sócio-educativa para Familiares e Cuidadores de Portadores da Doença de Alzheimer. Centro de Reabilitação e Hospital-dia Geriátrico do Instituto de Psiquiatria do Hospital das Clínicas. São Paulo, 2008.

FALCÃO, Deusivania Vieira da Silva. Doença de Alzheimer: um estudo sobre o papel das filhas cuidadoras e suas relações familiares. Brasilia: Universidade de Brasília, 2006. Tese de doutorado. Instituto de Psicologia.

GEERTZ, Clifford. A interpretação das culturas. Rio de Janeiro: Editora Guanabara, 1978. 
KARSH, Úrsula. Idosos Dependentes: famílias e cuidadores. Cadernos de Saúde Pública, Rio de Janeiro, v.19, n. 3, pp. 861-866, 2003.

NERI, Anita Liberalesso; SOMMERHALDER, Cínara. As várias faces do cuidado e do bem-estar do cuidador. In: NERI, Anita Liberalesso. (Org.). Cuidar de idosos no contexto da familia: questões psicológicas e sociais. Campinas, São Paulo: Alínea, 2002. p. 9-63

NOMURA, Shizuka., et al. Reabilitação Neuropsicológica. In: FORLENZA, Orestes; ALMEIDA, Paulo Neuropsiquiatria Geriátrica. São Paulo: Editora Atheneu, 2000, cap. 48, p. $539-547$

NORRIS, Margaret. Integrating families into Long-Term-Care Psychology Services: orchestrating cacophonies and symphonies. In: QUALLS, Sarah Hoon; ZARIT, Steven (Ed.) Aging families and caregiving. Hoboken, New Jersey: John Wiley \& Sons, Inc, 2009. p. 189-208.

QUEROZ, Nelma. Aspectos do conhecimento psicogerontológico para a atenção à família, ao cuidador e às instituições de idosos fragilizados. In: FALCÃO, Deusivania Vieira da Silva. (Org.) A Familia e o idoso: Desafios da contemporaneidade. Campinas, SP: Papirus, 2010, p. 111-128.

ZARIT, Steven. Empirically supported treatment for family caregivers. In: QUALLS, Sarah Honn; ZARIT, Steven. (Ed.) Aging families and caregiving. Hoboken, New Jersey: John Wiley \& Sons, Inc, 2009, p. 131-154.

\section{2) Ele foi consultado sobre essa decisão?}


3) Considera que foi a melhor decisão ou teria outra?

4) Como a sua família reagiu diante da decisão?

5) Como era o relacionamento do idoso com você antes da institucionalização?

6) Faz visitas? Com que freqüência?

7) Tem perspectivas de tirá-lo da instituição? 


\section{Questionário de percepção sobre \\ a Doença de Alzheimer}

\section{Instruções}

Este instrumento consiste numa série de questões a respeito de como você percebe a doença de Alzheimer, suas relações familiares e a institucionalização do seu familiar. É muito importante que você responda a todas elas, seguindo as instruções necessárias. Não há respostas certas ou erradas, podendo marcar mais de uma opção. O que importa é a sua sinceridade. Agradecemos, antecipadamente, sua valiosa colaboração.

Marque com X um ou mais itens das seguintes questões:

1) Para você o que é a doença de Alzheimer:

( ) Doença que provoca a perda da memória

( ) Doença crônica e degenerativa do cérebro

( ) Doença que deixa a pessoa desorientada

( ) doença que é desencadeada devido a fatores de hereditariedade

( ) Doença que não possui cura

( ) Doença que é desencadeada devido a características de personalidade

( ) Doença que é desencadeada devido a acontecimentos na vida do portador(a) ou da família (por exemplo, morte de parentes, acidentes, entre outros)

( ) Doença que é desencadeada devido a problemas espirituais

2) Como o Sr.(a) se sente com a institucionalização do seu familiar?

( ) Muito bem

( ) Bem

( ) Mais ou menos

( ) Mal

( ) Muito mal

3) Como o Sr.(a) define sua relação com seu familiar institucionalizado? 
4) O Sr.(a) considera que os encontros do grupo de familiares:

( ) Não são importantes para melhorar minha relação com meu familiar institucionalizado

( ) São pouco importantes para melhorar minha relação com meu familiar institucionalizado

( ) São mais ou menos importantes para melhorar minha relação com meu familiar institucionalizado

( ) São importantes para melhorar minha relação com meu familiar institucionalizado

( ) São muito importantes para melhorar minha relação com meu familiar institucionalizado

5) Por favor, complete as seguintes frases da maneira que desejar:

a) Eu considero que a ILPI

b) Participar do grupo

c) Na minha vida o grupo

d) Hoje eu me sinto

e) Para mim, a velhice é

f) O envelhecimento

Seu nome:

Nome do residente:

Anos de convívio com a doença:

Período de institucionalização: 